\title{
Management of a massive leiomyoma in pregnancy
}

\author{
Bernie McElhinney • Lathakutty Vinayakarao • \\ Raymond McClelland
}

Received: 14 June 2006 / Accepted: 22 August 2006 / Published online: 23 November 2006

(C) Springer-Verlag 2006

\begin{abstract}
This is a case report of a primigravida who was noticed to have a large pelvic mass at the time of her "booking" scan. A magnetic resonance imaging (MRI) scan was performed in order to elucidate the exact nature of the mass, which enlarged between successive scans. The appearances were in keeping with a leiomyoma undergoing degenerative change. The patient underwent a laparotomy in pregnancy to remove the leiomyoma, which was found to contain fluid-filled cavities. The uterine cavity was not breached at the time of surgery. The pregnancy continued uneventfully until term, and the baby was delivered by caesarean section. The postnatal period was uneventful.
\end{abstract}

Keywords Pregnancy $\cdot$ Complex mass · Leiomyoma ·

Laparotomy $\cdot$ Myomectomy

\section{Introduction}

Uterine leiomyomas are the most common type of benign neoplasm, with a reported incidence of $20-25 \%$ in the third and fourth decade [1]. Although the precise aetiology is unknown, it is clear that hormones play a pivotal role [2]. Uterine leiomyoma occurred approximately $0.1 \%$ of the time in pregnant patients [3], with a reported range of $0.2-7.2 \%$ [4]. One of the major complications in pregnancy is pain

B. McElhinney $(\varangle) \cdot$ R. McClelland

Belfast City Hospital,

Lisburn Road,

Belfast BT9 7AB, Ireland

e-mail: b.mcelhinney@qub.ac.uk

L. Vinayakarao

Craigavon Area Hospital,

66 Lurgan Road,

Portadown, Craigavon BT63 5QQ, Ireland secondary to degeneration of the mass, which usually occurs between the 14th and 20th week when the growth of the uterus is most active [3]. After delivery, the majority of leiomyomas regress. Complications, such as spontaneous abortion, premature labour, soft tissue dystocia, uterine inertia, foeto-pelvic disproportion, foetal malposition, retained products and postpartum haemorrhage have all been documented [5]. Gestational leiomyomas can have an unusual appearance that may be misinterpreted and mistaken for malignancy. In this report, we describe the case of a patient with an incidental uterine leiomyoma in pregnancy, which underwent massive enlargement in the absence of symptoms.

\section{Case report}

A 29-year-old para $0^{+0}$ booked for antenatal care at 9 weeks amenorrhoea. An ultrasound scan revealed a singleton, active foetus with biometry appropriate for dates. A large mass of undetermined origin was noted adjacent to the gravid uterus. Serum cancer antigen (CA-125) level was checked to rule out ovarian pathology and was slightly elevated, with a level of $55 \mathrm{u} / \mathrm{l}$. There was no past gynaecological history of note; a routine smear 4 years previously had been reported as normal. The patient was reviewed at 11 weeks' gestation, and a further ultrasound scan confirmed the presence of a large, mixed echogenic mass arising from the pelvis (Figs. 1 and 2). A magnetic resonance imaging (MRI) examination was arranged, and the report described a multi-loculated and cystic pelvic mass extending into the abdomen. The mass measured $16 \mathrm{~cm}$ in maximum transverse diameter and $18 \mathrm{~cm}$ in maximum cranio-caudal length (Figs. 3 and 4), and it appeared to arise from the anterior wall of the uterus.

An oncology opinion was sought in view of the rapid growth and unusual appearance of the mass, and a decision 


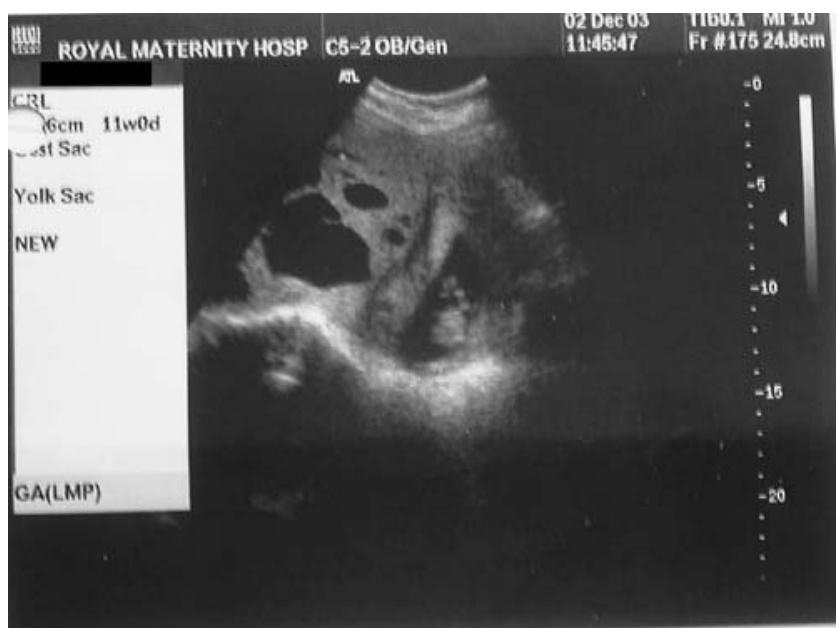

Fig. 1 Real-time scan image at booking visit demonstrating complex adnexal mass adjacent to viable intra-uterine pregnancy (IUP)

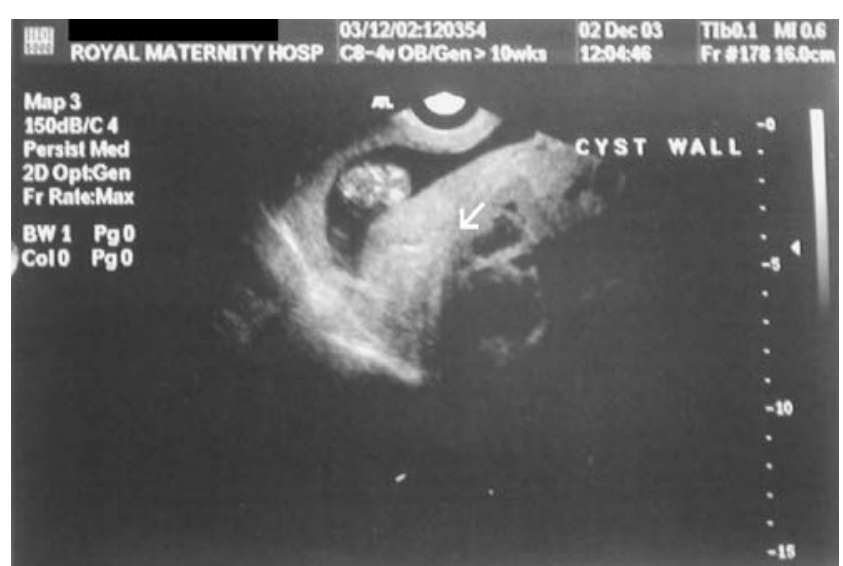

Fig. 2 Further image of complex adnexal mass adjacent to viable intra-uterine pregnancy (IUP)

was made to perform an exploratory laparotomy. At 15 weeks' gestation, the patient was admitted to the oncology ward. On examination, the abdomen was soft and non-tender, and fundal height was midway between the umbilicus and xiphisternum. A routine mid-line laparotomy was performed, and a 34-week-size uterus was evident. The gravid uterus lay posteriorly, and a large leiomyoma arose from the anterior surface. A survey of the pelvis and abdomen was normal, and washings were obtained for cytological analysis. Both ovaries were entirely normal and mobile. A syringe was inserted into the fibroid mass, and $700 \mathrm{ml}$ of clear yellow fluid was aspirated. An incision was made in the superior aspect of the uterus to facilitate enucleation of a large, singular, complex mass (Figs. 5 and 6). Excess uterine tissue was excised, and the "dead space" closed with three-layer technique, with a good cosmetic result (Figs. 7 and 8). Intra-operatively, the uterine cavity remained intact.

The post-operative period was uneventful, and ultrasound scan revealed a viable foetus. The patient was discharged home on day 7 , and histological examination was reported as "cystic degeneration of a fibroid".

\section{Discussion}

Uterine leiomyomas occur during the reproductive period, a time when hormonal influences are at their maximum [2]. Leiomyomas are hypersensitive to oestrogen, and several studies have demonstrated a significantly greater number of oestrogen receptors in leiomyomas than in surrounding normal myometrium $[6,7]$. It is well known that oestrogen levels rise steadily throughout pregnancy; however, serial scans have indicated that continuous growth of a leiomyoma throughout pregnancy is unusual and documented in only a small fraction of cases (0.06\%) [7]. Complications occur almost exclusively in large lesions during pregnancy, delivery and the puerperium [8]; the majority of pregnancies having good outcomes [7].

The larger the leiomyoma, the more likely that some form of degeneration will be present. The type of degeneration depends on the degree and rapidity of the onset of vascular insufficiency caused by the enlarging leiomyoma outgrowing its blood supply. The most common form is hyaline degeneration, which usually undergoes liquefaction with the formation of cystic spaces without epithelial linings. These

Fig. 3 Magnetic resonance image (MRI) of lesion demonstrating uterine origin and solid/ cystic components

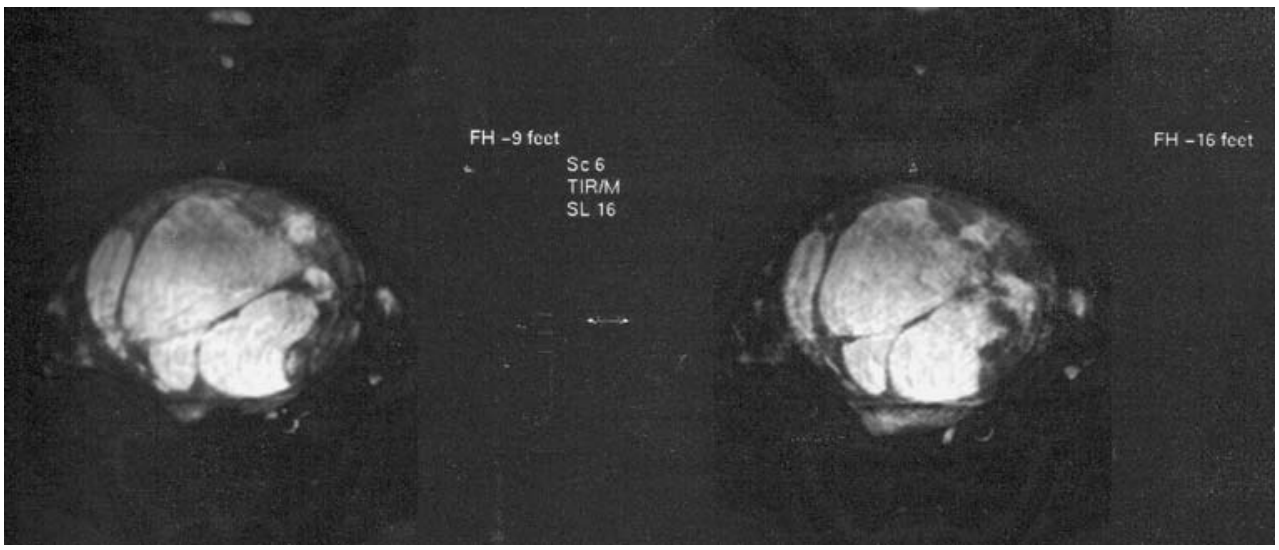


Fig. 4 Longitudinal sagittal view of gravid uterus with appearances in keeping with a degenerating fibroid

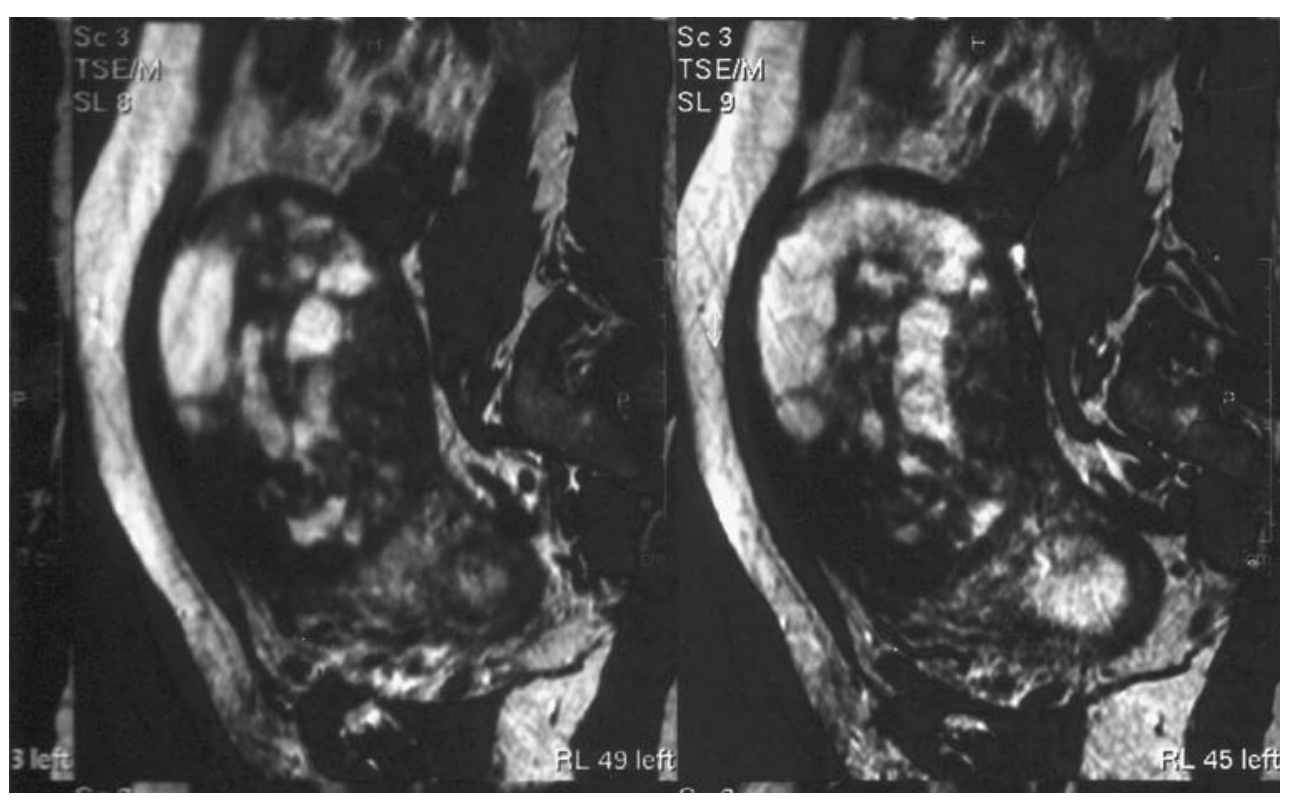

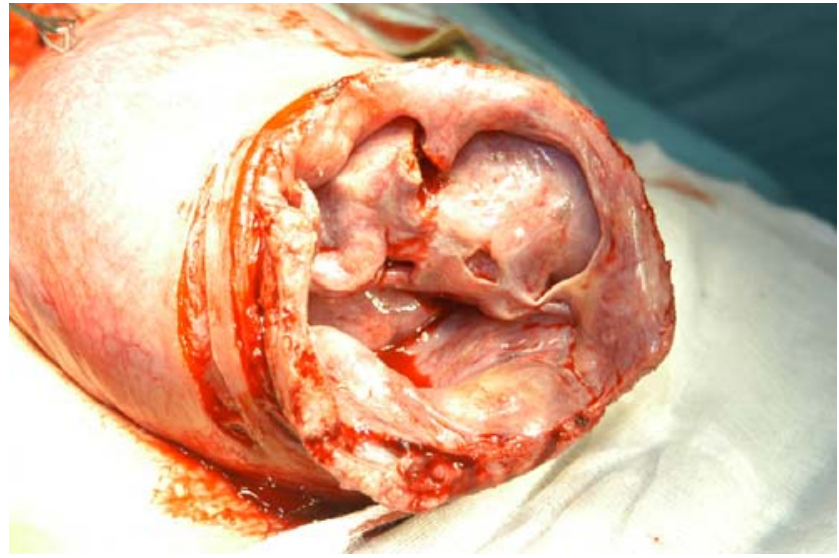

Fig. 5 Intra-operative view demonstrating "cut" surface of degenerating fibroid in gravid uterus

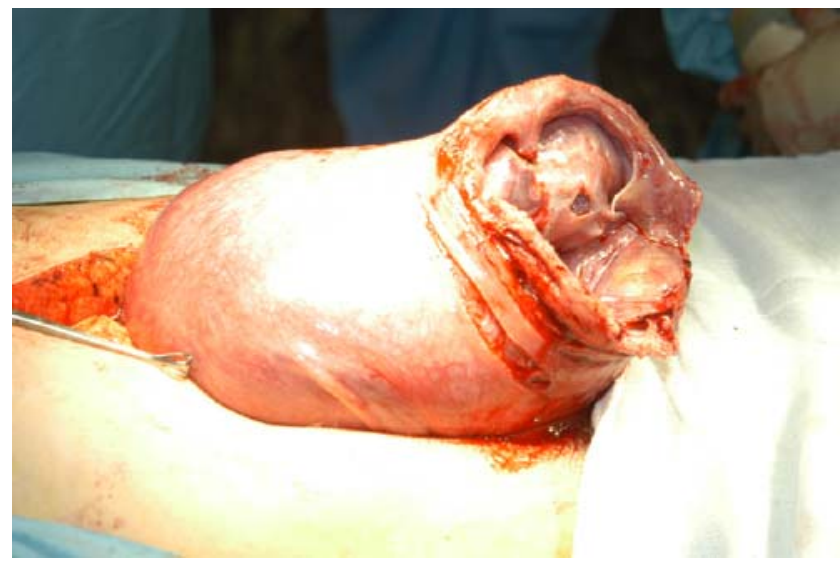

Fig. 6 Longitudinal view of gravid uterus after incision of superior aspect of degenerating fibroid

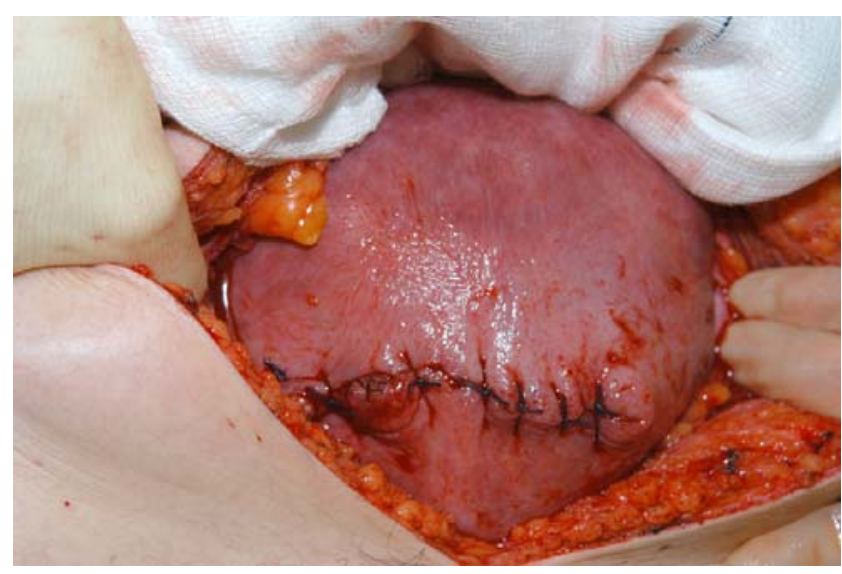

Fig. 7 Post-operative appearance of uterus after removal of fibroid

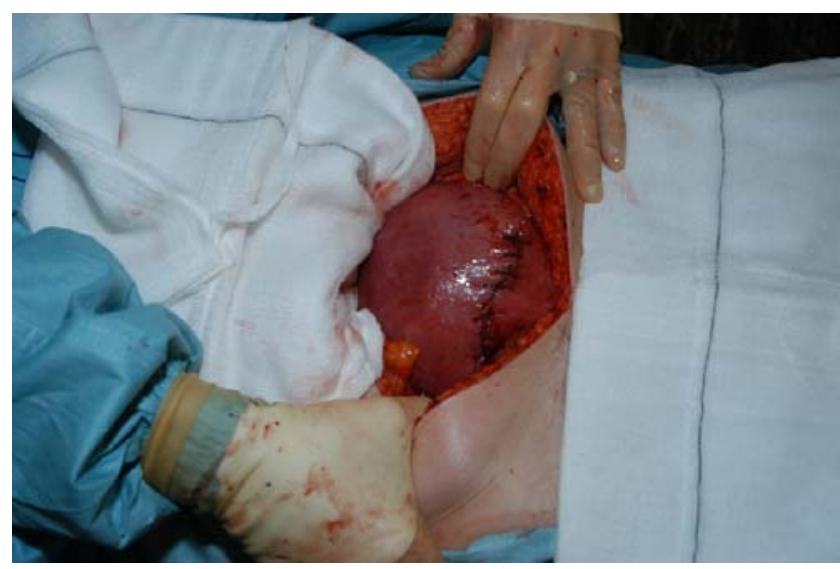

Fig. 8 Gravid uterus is replaced in the pelvic cavity 
cystic areas are filled with a colourless or blood-stained fluid. Red degeneration results from haemorrhagic infarction and occurs in approximately $8 \%$ of leiomyomas, complicating pregnancy. The process is often the cause of pain, fever and low-grade leukocytosis [9]. The degree of degeneration does not necessarily correlate with the severity of symptoms, and in this case, there was no history of symptomatology suggestive of degeneration.

The differential diagnosis of a pelvic mass includes ovarian, cervical or endometrial neoplasm and uterine leiomyoma with or without sarcomatous change. In this case, the unusual radiological appearance and rapid growth were of concern. Rapid growth of a leiomyoma is not a reliable indication of sarcomatous degeneration. In a retrospective review of 580 leiomyosarcomas, less than $3 \%$ of patients had a rapidly enlarging uterus. In the same review, only one leiomyosarcoma was found in 371 women operated on for rapidly growing leiomyomas [10].

MRI is increasingly being utilised in the assessment of gynaecological pelvic masses. MRI was found to be the most accurate imaging technique for detection and localisation of leiomyomas [1] and would appear to be superior to ultrasound in the precise imaging of indeterminate solid pelvic masses. Kier et al. [11] reported on the assessment of 17 patients with ultrasonographically diagnosed pelvic masses by MRI. These authors noted that there was $100 \%$ accuracy in identifying the organ of origin on MRI. Sherer et al. [9] reviewed the literature and reported on a case demonstrating the usefulness of MRI in the precise diagnosis of an undetermined mass in pregnancy.

In our case, the patient was referred to the oncology department for further assessment. An MRI was arranged that, in coronal and sagittal planes, depicted a band of tissue surrounding both the pregnancy and the mass, in keeping with an intra-mural location, as would be found with a leiomyoma. This led to a presumptive diagnosis of leiomyoma with degenerative change, and surgery was planned as an elective procedure. Surgery is rarely necessary, but it was essential in this case due to the massive enlargement of the leiomyoma and potential pregnancy-related problems.

Hasan et al. [3] presented 60 cases of uterine leiomyomas in pregnancy. The overall incidence was $0.1 \%$, and the median age was 33.4 (range 22-45) years. A pre-natal history of leiomyomas was present in $45 \%$ of cases; $35 \%$ were diagnosed at the booking visit, $13.3 \%$ at delivery and the remainder were discovered at the time of laparotomy for a suspected ovarian accident. In $10 \%$ of cases, symptoms suggestive of red degeneration were recorded; $73 \%$ of patients were delivered by caesarean delivery, and ten patients experienced severe haemorrhage, 3 of whom required a caesarean hysterectomy. In certain incidences, a hysterectomy may be life saving, and it is important that the patient is properly counselled regarding this.
Surgery is also indicated when other complications cannot be ruled out, such as ovarian accident, appendicitis or placental abruption. Varras et al. [12] reported on a case of intra-peritoneal haemorrhage secondary to perforation of a uterine fibroid after cystic degeneration, resulting in a sub-total hysterectomy. They concluded that the diagnosis of ruptured cystic degeneration of uterine leiomyoma should be considered in the presence of an acute abdomen and a pelvic mass; an exploratory laparotomy is mandatory. The authors remarked that the first reported case was an autopsy finding (1867), and even today, precise preoperative diagnosis is uncommon.

Our case is an interesting presentation of a rapidly enlarging gestational leiomyoma in the absence of symptoms. The dramatic appearances on ultrasound scan and MRI were secondary to hyaline degeneration. This case sets an example for the usefulness of MRI in the diagnosis of an undetermined mass in pregnancy. The patient suffered no ill-effects from surgery, and the pregnancy continued until term, when she had a caesarean delivery of a healthy male infant.

\section{References}

1. Murase E, Siegelman E, Outwater E, Perez-Jaffe L, Tureck R (1999) Uterine leiomyomas: histopathologic features, MR imaging findings, Differential diagnosis, and treatment. Radiographics 19:1179-1197

2. Robboy S, Bentley R, Butnor K, Anderson M (2000) Pathology and Pathophysiology of uterine smooth-muscle tumours. Environ Health Perspect 108:779-784

3. Hasan F, Arumugam K, Sivanesaratnam V (1990) Uterine Leiomyomata in pregnancy. Int J Gynaecol Obstet 34:45-48

4. Gainey H, Keeler J (1949) Submucous myoma in term pregnancy. Am J Obstet Gynecol 58:727

5. Mason T (2002) Red degeneration of a leiomyoma masquerading as retained products of conception. J Natl Med Assoc 94:124-126

6. Andersen J, Barbieri R (1995) Abnormal gene expression in uterine leiomyomas. J Soc Gynecol Investig 2:663-672

7. Simon F (1988) Leiomyomas in Pregnancy. Am Fam Physician $37: 163-166$

8. Huch Boni R, Hebisch G, Huch A, Stallmach T, Krestin G (1994) Multiple necrotic uterine leiomyomas causing severe puerperal fever: Ultrasound, CT, MR, and histological findings. J Comput Assist Tomogr 18:828-831

9. Sherer D, Maitland C, Levine N, Eisenberg C, Abulafia O (2000) Prenatal magnetic resonance imaging assisting in differentiating between large degenerating intramural leiomyoma and complex adnexal mass during pregnancy. J Matern Fetal Med 9:186-189

10. Parker W, Fu Y, Berek J (1994) Uterine sarcoma in patients operated on for presumed leiomyoma and rapidly growing leiomyoma. Obstet Gynecol 83:414-418

11. Kier R, McCarthy S, Scoutt L, Viscarello R, Schwartz P (1990) Pelvic masses in pregnancy: MR imaging. Radiology 176:709-713

12. Varras M, Antoniou S, Samara C, Frakala S, Angelidou-Manika Z, Paissios P (2002) Intraperitoneal haemorrhage secondary to perforation of uterine fibroid after cystic degeneration. Unusual CT findings resembling malignant pelvic tumour: case report. Eur J Gynaecol Oncol 23:565-568 